\title{
Tratamento dos transtornos alimentares: fatores associados ao abandono
}

\author{
Eating disorders treatment: factors associated with dropout \\ Ana Paula Leme de Souza', Rosane Pilot Pessa²
}

\section{RESUMO}

Objetivos: Traçar o perfil do abandono do tratamento de pacientes com transtornos alimentares (TA) em um serviço especializado e investigar os fatores associados. Métodos: Estudo transversal com delineamento quantitativo do tipo comparativo. Todos os prontuários de pacientes atendidos pelo serviço, entre 1982 e 2013, foram revisados para coletar dados sociodemográficos, clínicos e antropométricos (no primeiro e último atendimento), e resultado do tratamento. Os dados foram analisados pelo programa SPSS e R. Foram utilizados testes qui-quadrado de Pearson, exato de Fisher, não paramétrico de Mann-Whitney e análise de regressão logística. Resultados: Dos pacientes, 66,7\% abandonaram o tratamento (Grupo Abandono - GA) e 33,3\% tiveram outros resultados (Grupo Não Abandono - GNA). No GA, a maioria era do sexo feminino, de Ribeirão Preto e região, estudantes, solteiros, com escolaridade mínima do nível fundamental e com anorexia nervosa (AN). Houve associação significativa com o abandono nas variáveis Hipótese Diagnóstica $(p=0,049)$, Comorbidades Psiquiátricas ( $p=0,001)$, Depressão $(p=0,048)$, Transtornos de Personalidade $(p=0,001)$, Comorbidades Clínicas $(<0,001)$ e Osteopenia $(p=0,007)$. No GA, eles tinham peso adequado e ausência de amenorreia, tanto no início quanto no final do seguimento. O estado nutricional adequado e a ausência de comorbidades clínicas se associaram com o abandono.

\section{Palavras-chave}

Transtornos alimentares, resultado do tratamento, pacientes desistentes do tratamento.
Conclusões: A taxa de abandono do serviço é alta e pacientes nessa condição eram adultos jovens, tinham diagnóstico de AN, longo tempo de sintomas antes do início do tratamento e estavam há menos de seis meses no seguimento. Estudos prospectivos poderão contribuir para pesquisas dirigidas ao abandono do seguimento desses pacientes, buscando melhor compreensão dessas doenças e seu tratamento.

\section{ABSTRACT}

Objectives: To outline the profile of patients with eating disorders who drop out of a specialized service treatment and to investigate the associated factors. Methods: Cross-cut study with a comparative quantitative outline. All patients' medical records being treated by the service, between 1982 and 2013, have been reviewed in order to collect social and demographic data, as well as clinical and anthropometrical data (in the first and last medical visit) and data related to the outcome of the treatment. Data has been analyzed by SPSS and R program and the following tests have been used: Pearson's chi-square, Fisher's exact test, Mann-Whitney $U$ test and logistic regression analysis. Results: Sixty-six point seven percent of patients

1 Universidade de São Paulo (USP), Escola de Enfermagem de Ribeirão Preto; Grupo de Assistência em Transtornos Alimentares (GRATA). 2 USP, Escola de Enfermagem de Ribeirão Preto, Departamento de Enfermagem Materno-Infantil e Saúde Pública.

Endereço para correspondência: Ana Paula Leme de Souza Rua Sargento Bueno, 101, Torre 2, ap. 1201, Parque dos Lagos 14093-017 - Ribeirão Preto, SP E-mail: apls.nutri@gmail.com 


\section{Keywords}

Eating disorders, treatment

outcome, patient dropouts. dropped out of treatment (Dropout Group-DG) and 33.3\% had different outcomes (Persisters Group-PG) Among DG, most were female, from Ribeirão Preto and surroundings, student, single, at least with Junior and Elementary High School and a diagnosis of anorexia nervosa (AN). Dropout was materially associated with the following variables Diagnostic Hypothesis $(p=0.049)$, Psychiatric Comorbidities $(p=0.001)$, Depression $(p=0.048)$, Personality Disorder $(p=0.001)$, Clinical Comorbidities ( $<0.001)$ and Osteopenia $(p=0.007)$. Among DG, subjects had appropriate weight and absence of amenorrhea both at the beginning and end of the treatment. Good nutritional status and absence of clinical comorbidities were associated with dropout. Conclusions: The dropping out rate of the service is high. Patients were young adults diagnosed with AN who have long displayed symptoms before the beginning of the treatment and were less than six months under treatment. Prospective studies can contribute to researches driven to the dropout of treatment by these patients, researches which could seek and provide a better understanding of these disorders and how to treat them.

\section{INTRODUÇÃO}

Os transtornos alimentares (TA) são doenças mentais que abrangem sintomas físicos e psíquicos ${ }^{1}$ cuja etiologia é multifatorial, envolvendo predisposição genética e sociocultural, vulnerabilidade biológica e psicológica, além de questões familiares de padrões de vínculo disfuncionais ${ }^{2,3}$.

Tanto a anorexia nervosa (AN) quanto a bulimia nervosa (BN) e o transtorno alimentar sem outra especificação (TASOE) envolvem comportamentos alimentares desorganizados e desequilibrados, além de distorção da imagem corporal $^{4,5}$. São caracterizados por recaídas frequentes e o seguimento, na maioria dos casos, é longo e extremamente trabalhoso. O tratamento objetiva a completa reabilitação do paciente nos aspectos clínicos, nutricionais e psicológicos, e o trabalho em equipe multidisciplinar com estrutura básica formada por médicos psiquiatra e clínico geral ou nutrólogo, nutricionista e psicólogo é reconhecido como a forma mais adequada de acompanhamento ${ }^{5,6}$.

O sucesso de um programa de atendimento integrado e completo depende da existência desse time, além do emprego conjunto de diversas estratégias. Porém, nenhuma modalidade de tratamento pode ser indicada como única ou isoladamente melhor. Essa equipe funciona como uma rede de sustentação para o paciente que descobre, nos vários profissionais, a possibilidade de diversas transferências, tornando-se mais amplas as possibilidades de adesão ao tratamento ${ }^{7,8}$.

Os serviços especializados para o tratamento podem ser encontrados em unidades de clínica médica, pediátrica e psiquiátrica, e as modalidades de seguimento dependerão da avaliação das condições clínicas, psiquiátricas e de apoio familiar para o seguimento do paciente e variam entre atendimento ambulatorial, hospital-dia e internação integral ${ }^{9}$. Se o tratamento não se inicia de forma precoce, o curso é prolongado, com prejuízos físicos e psicológicos, além de elevada taxa de mortalidade, principalmente em pacientes com AN ${ }^{10}$.
Aliado a essa questão, pacientes com TA tendem a negar a doença e usam com frequência racionalizações para justificar sua sintomatologia. Muitas vezes, declaram tratar-se de um estilo de vida que resolveram seguir, uma opção que tomaram conscientemente para lidar com suas preocupações com o corpo e, por consequência, minimizam os riscos potenciais aos quais se expõem, inclusive de morte. Consequentemente, negam também o tratamento, principalmente no período inicial quando ainda não se formaram as bases seguras que geram o fortalecimento da aliança terapêutica. Nesses casos, os profissionais devem manter atenção redobrada ao tipo de vínculo que o paciente reproduz no ambiente de tratamento, procurando desfazer as distorções que incrementam a desconfiança que eles geralmente apresentam com relação à qualidade e à consistência de tudo que recebem do outro ${ }^{11}$.

Devido à sua etiologia multifatorial, uma clara delimitação dos fatores de risco envolvidos, da evolução do quadro e dos aspectos que favorecem a recuperação é difícil de ser obtida ou é imprecisa. Estudos prospectivos poderiam revelar, de forma mais objetiva, o curso desses transtornos, porém esses são de difícil manejo, de longo prazo, mais onerosos e com maior risco de perdas amostrais ${ }^{7}$. Na literatura, há consenso geral de que os tratamentos disponíveis para os principais transtornos psiquiátricos frequentemente resultam na ausência de efeito ou numa resposta moderada a ele ${ }^{12}$.

Além disso, os critérios e procedimentos para avaliação do prognóstico dos TA variam muito. Os mais utilizados são os critérios da Escala de Categorias de Desfecho Geral de Morgan-Russel, que classifica a evolução do TA em favorável, intermediária e desfavorável, de acordo com o peso e ciclo menstrual para AN, e peso e sintomas de compulsão e purgação para a BN, avaliando a condição geral do paciente (estado nutricional e mental, nível socioeconômico e ajustamento psicossocial) ${ }^{13}$.

Dessa forma, o tratamento mostra-se desafiador em todas as fases da doença, pois os pacientes mostram-se des- 
confiados, resistentes às condutas adotadas e frequentemente não aderem ao esquema terapêutico proposto ${ }^{14,15}$. O abandono do tratamento representa risco físico e psicológico ao paciente, além de impactos financeiros aos serviços de saúde cujas razões ainda não são claramente identificadas ${ }^{15}$. Poucos são os estudos que investigaram essa temática, e os resultados mostram altas taxas de abandono. Fassino et al. (2009), em revisão de literatura que incluiu 26 estudos, dos quais três foram randomizados e controlados e 23 retrospectivos não controlados, e com amostra de 20 a 261 indivíduos, observaram que $20,2 \%$ a $51 \%$ dos pacientes internados e $29 \%$ a $73 \%$ dos pacientes ambulatoriais, respectivamente, abandonaram 0 tratamento ${ }^{16}$. Esses dados sugerem que mais pesquisas devem ser desenvolvidas visando elucidar os principais motivos desse fenômeno, com vistas a subsidiar o aprimoramento dos protocolos de assistência, trazendo resultados terapêuticos mais efetivos ${ }^{17-19}$.

Nesse contexto, o objetivo deste estudo foi traçar o perfil do abandono do tratamento de pacientes com TA em um serviço especializado e investigar os fatores associados.

\section{MÉTODOS}

Trata-se de estudo descritivo, transversal com delineamento quantitativo do tipo comparativo, desenvolvido em um serviço especializado para tratamento de TA no interior do estado de São Paulo, Brasil20-22. Os participantes foram todos os pacientes atendidos por esse serviço, independentemente da modalidade de atendimento (ambulatorial e internação integral) desde sua criação, em 1982, até dezembro de 2013.

Os dados foram coletados a partir do levantamento dos prontuários dos pacientes com diagnóstico de AN, BN ou TASOE que foram atendidos pelo serviço, por meio do sistema informatizado do hospital no Departamento de Seção de Dados Médicos. Foram excluídos do estudo aqueles pacientes que, apesar do diagnóstico do TA, não receberam atendimento pelo serviço ou cujos prontuários não foram encontrados nos arquivos médicos. Com base nesses critérios, realizou-se revisão de todos os arquivos disponíveis (prontuário físico, digitalizado e microfilmado) com o intuito de verificar o seguimento do paciente no momento inicial do tratamento (primeiro atendimento), e em seu último retorno ao serviço (último atendimento). As variáveis escolhidas para investigação foram as que geralmente são descritas nos prontuários do serviço: características sociodemográficas; diagnóstico do TA; comorbidades psiquiátricas, considerando qualquer diagnóstico psiquiátrico; e comorbidades clínicas, considerando qualquer diagnóstico clínico. Foram analisados, separadamente, os diagnósticos psiquiátricos e clínicos que apareceram com maior frequência (transtornos de personalidade e depressão, alterações digestivas, distúr- bios hidroeletrolíticos, anemias, osteopenia e osteoporose); dados antropométricos (peso e altura para cálculo do índice de massa corporal - IMC); época do atendimento e presença de amenorreia (no primeiro e último atendimento) e resultado do tratamento no serviço (óbito, alta, encaminhamento para outros serviços, em seguimento no momento da coleta de dados e abandono). Os critérios de alta adotados pelo serviço incluem a normalização do peso, padrão alimentar adequado, ausência de distorção de imagem corporal e quadros clínico, psiquiátrico e emocional estáveis. Os encaminhamentos para outros serviços de saúde ocorrem por questões de distância geográfica e dificuldade de seguimento mais frequente, e quando o TA é secundário a outros transtornos psiquiátricos. O abandono do tratamento foi definido como a decisão unilateral do paciente para interromper o acompanhamento sem o consentimento da equipe terapêutica. Portanto, foram considerados pacientes nessa situação aqueles que não foram a óbito, não receberam alta, não foram encaminhados para outros serviços de saúde e não estavam em seguimento no momento da coleta de dados.

Os dados foram analisados de forma descritiva pelos programas SPSS versão 22 e R versão 3.0.2. Para as variáveis categóricas, utilizaram-se os testes Qui-Quadrado de Pearson e o teste exato de Fisher. Para as variáveis numéricas, foi utilizado o teste não paramétrico de Mann-Whitney. Posteriormente, realizou-se regressão logística considerando a variável Abandono como dependente, e como variáveis independentes aquelas que apresentaram significância estatística nos testes de associação e comparação.

Esta pesquisa foi aprovada pelo Comitê de Ética em Pesquisa da Escola de Enfermagem de Ribeirão Preto, da Universidade de São Paulo (Parecer no 216/2013).

\section{RESULTADOS}

Foram identificados 243 prontuários de pacientes atendidos pelo serviço; desses, nove não foram localizados, totalizando a amostra em 234 pacientes. Os seguintes resultados foram encontrados: $156(66,7 \%)$ pacientes abandonaram o tratamento (Grupo Abandono - GA) e 78 (33,3\%) tiveram outros resultados (Grupo Não Abandono - GNA): alta ( $n=47$; $60,3 \%$ ), óbito ( $n=02 ; 2,6 \%)$, encaminhamento para outros serviços ( $n=8 ; 10,2 \%)$ e em seguimento no momento da coleta de dados $(n=21 ; 26,9 \%)$.

Em relação às variáveis sociodemográficas investigadas, não houve diferença significativa entre os grupos GA e GNA (Tabela 1).

No entanto, houve diferença entre os grupos nas variáveis Hipótese Diagnóstica, Comorbidades Psiquiátricas, Depressão, Transtornos de Personalidade, Comorbidades Clínicas e Osteopenia (Tabela 2). 
Tabela 1. Dados sociodemográficos dos participantes do estudo de acordo com o desfecho do tratamento - Ribeirão Preto-SP, 2013

\begin{tabular}{|c|c|c|c|}
\hline & $\begin{array}{c}\text { Grupo } \\
\text { Abandono- } \\
\text { GA }(n=156)\end{array}$ & $\begin{array}{l}\text { Grupo Não } \\
\text { Abandono- } \\
\text { GNA }(n=78)\end{array}$ & $p$ \\
\hline Sexo & & & 0,756 \\
\hline Feminino & $140(66,4 \%)$ & $71(33,6 \%)$ & \\
\hline Masculino & $16(69,6 \%)$ & $07(30,4 \%)$ & \\
\hline Procedência & & & 0,449 \\
\hline Ribeirão Preto e região & $120(66,7 \%)$ & $60(33,3 \%)$ & \\
\hline Outras cidades de São Paulo & $23(71,9 \%)$ & $09(28,1 \%)$ & \\
\hline Outros estados & $11(55 \%)$ & 09 (45\%) & \\
\hline Escolaridade & & & 0,674 \\
\hline $\begin{array}{l}\text { Ensino fundamental incompleto ou } \\
\text { analfabeto }\end{array}$ & $29(67,4 \%)$ & $14(32,6 \%)$ & \\
\hline Ensino fundamental completo & $45(68,2 \%)$ & $21(31,8 \%)$ & \\
\hline Ensino médio completo & $32(58,2 \%)$ & $23(41,8 \%)$ & \\
\hline Ensino superior completo & $17(63 \%)$ & $10(37 \%)$ & \\
\hline Profissão & & & 0,643 \\
\hline Estudante & $86(65,2 \%)$ & $46(34,8 \%)$ & \\
\hline Do lar & $13(69,4 \%)$ & $06(31,6 \%)$ & \\
\hline Profissão com ensino superior & $16(80 \%)$ & $04(20 \%)$ & \\
\hline Outras profissões & $24(64,9 \%)$ & $13(35,1 \%)$ & \\
\hline Aposentado, desempregado ou inativo & $06(54,5 \%)$ & $05(45,5 \%)$ & \\
\hline Estado civil & & & 0,535 \\
\hline Solteiro & $122(65,9 \%)$ & $63(34,1 \%)$ & \\
\hline Casado & $28(71,8 \%)$ & $11(28,2 \%)$ & \\
\hline Separado/divorciado & $03(50 \%)$ & $03(50 \%)$ & \\
\hline
\end{tabular}

No que se refere aos dados do atendimento clínico, a maioria dos pacientes que abandonaram o tratamento (GA) recebeu o primeiro e o último atendimento nos anos 2000. O estado nutricional foi de eutrofia com ausência de amenorreia no momento inicial e final do seguimento. O estado nutricional apresentou associação significativa com o resultado do tratamento, sugerindo que pacientes com eutrofia no período inicial do tratamento possuem maiores chances de abandonar o seguimento (Tabela 3).

Os pacientes dos dois grupos (GA e GNA) eram adultos jovens que apresentavam longo tempo de sintomas antes do início do tratamento (Tabela 4). Em relação ao estado nutricional, a diferença entre os grupos foi no primeiro atendimento, ao se observar que pacientes do GA eram eutróficos, enquanto os do GNA apresentavam magreza. Nota-se também que o tempo de amenorreia foi muito variável em ambos os grupos.

O tempo de tratamento no serviço de ambos os grupos pode ser observado na Tabela 5. Nota-se que a maioria dos pacientes que abandonaram o seguimento o fez de maneira precoce, antes dos seis primeiros meses de tratamento. Já aqueles que não abandonaram tiveram acompanhamento superior a 12 meses.
Tabela 2. Diagnósticos psiquiátricos e clínicos dos participantes do estudo no início do tratamento - Ribeirão Preto-SP, 2013

\begin{tabular}{|c|c|c|c|}
\hline & $\begin{array}{c}\text { Grupo } \\
\text { Abandono- } \\
G A(n=156)\end{array}$ & $\begin{array}{l}\text { Grupo Não } \\
\text { Abandono - } \\
\text { GNA ( } n=78)\end{array}$ & $\mathrm{p}$ \\
\hline Hipótese diagnóstica & & & 0,049 \\
\hline Anorexia nervosa & $103(62,0 \%)$ & $63(38,0 \%)$ & \\
\hline Bulimia nervosa & $41(80,4 \%)$ & $10(19,6 \%)$ & \\
\hline $\begin{array}{l}\text { Transtorno alimentar sem outra } \\
\text { especificação }\end{array}$ & $12(70,6 \%)$ & $05(29,4 \%)$ & \\
\hline Comorbidades psiquiátricas & & & 0,001 \\
\hline Sim & $61(56,0 \%)$ & $48(44,0 \%)$ & \\
\hline Não & $95(76,0 \%)$ & $30(24,0 \%)$ & \\
\hline Depressão & & & 0,048 \\
\hline Sim & $30(55,6 \%)$ & $24(44,4 \%)$ & \\
\hline Não & $126(70,0 \%)$ & $54(30,0 \%)$ & \\
\hline Transtornos de personalidade & & & 0,001 \\
\hline Sim & $39(52,0 \%)$ & $36(48,0 \%)$ & \\
\hline Não & $117(73,6 \%)$ & $42(26,4 \%)$ & \\
\hline Comorbidades clínicas & & & $<0,001$ \\
\hline Sim & $33(49,3 \%)$ & $34(50,7 \%)$ & \\
\hline Não & $123(73,7 \%)$ & $44(26,3 \%)$ & \\
\hline Alterações digestivas & & & 0,367 \\
\hline Sim & $07(53,8 \%)$ & $06(46,2 \%)$ & \\
\hline Não & $149(67,4 \%)$ & $72(32,6 \%)$ & \\
\hline Distúrbios hidroeletrolíticos & & & 0,403 \\
\hline Sim & $03(50,0 \%)$ & $03(50,0 \%)$ & \\
\hline Não & $153(67,1 \%)$ & $75(32,9 \%)$ & \\
\hline Anemias & & & 1,0 \\
\hline Sim & $05(62,5 \%)$ & $03(37,5 \%)$ & \\
\hline Não & $151(66,8 \%)$ & $75(33,2 \%)$ & \\
\hline Osteopenia & & & 0,007 \\
\hline Sim & $02(22,2 \%)$ & $07(77,8 \%)$ & \\
\hline Não & $154(68,4 \%)$ & $71(31,6 \%)$ & \\
\hline Osteoporose & & & 0,187 \\
\hline Sim & $05(45,5 \%)$ & $06(54,5 \%)$ & \\
\hline Não & $151(67,7 \%)$ & $72(32,3 \%)$ & \\
\hline
\end{tabular}

Com a estimativa dos parâmetros do modelo de regressão logística (Tabela 6), observou-se que a variável Comorbidades Clínicas foi a única que apresentou associação com o abandono do tratamento.

A regressão logística considerando a variável Abandono como dependente, e como variáveis independentes aquelas que apresentaram significância estatística nos testes de associação e comparação, revelou que os pacientes que iniciaram o tratamento na primeira década de atuação do serviço (1980-1989) possuem mais chances de abandonar o tratamento do que aqueles que tiveram o primeiro atendimento entre os anos 2010 e 2013, considerando o mesmo nível de comorbidades clínicas, tempo de tratamento e estado nutricional. O mesmo comportamento foi observado para aqueles pacientes com tempo de tratamento inferior a seis meses e com excesso de peso (Tabela 7). 
Tabela 3. Dados do atendimento clínico e da doença dos participantes do estudo segundo o momento (primeiro e último atendimento) - Ribeirão Preto-SP, 2013

\begin{tabular}{|c|c|c|c|}
\hline & $\begin{array}{c}\text { Grupo } \\
\text { Abandono } \\
-G A \\
(n=156)\end{array}$ & $\begin{array}{c}\text { Grupo Não } \\
\text { Abandono } \\
\text {-GNA } \\
(\mathrm{n}=78)\end{array}$ & $\mathbf{p}$ \\
\hline Época do $1^{0}$ atendimento & & & 0,002 \\
\hline Anos 1980 & $16(76,2 \%)$ & $5(23,8 \%)$ & \\
\hline Anos 1990 & $44(67,7 \%)$ & $21(32,3 \%)$ & \\
\hline Anos 2000 & $85(72,0 \%)$ & $33(28,0 \%)$ & \\
\hline Anos 2010 & $11(36,7 \%)$ & $19(63,3 \%)$ & \\
\hline Época do último atendimento & & & $<0,001$ \\
\hline Anos 1980 & $10(76,9 \%)$ & $03(23,1 \%)$ & \\
\hline Anos 1990 & $43(81,1 \%)$ & $10(18,9 \%)$ & \\
\hline Anos 2000 & $89(76,1 \%)$ & $28(23,9 \%)$ & \\
\hline Anos 2010 & $14(27,5 \%)$ & $37(72,5 \%)$ & \\
\hline Estado nutricional no $1^{\circ}$ atendimento & & & 0,004 \\
\hline Eutrofia & $85(75,9 \%)$ & $27(24,1 \%)$ & \\
\hline Magreza & $58(55,2 \%)$ & $47(44,8 \%)$ & \\
\hline Excesso de peso & $13(76,5 \%)$ & $04(23,5)$ & \\
\hline Estado nutricional no último atendimento & & & 0,625 \\
\hline Eutrofia & $104(68,4 \%)$ & $48(31,6 \%)$ & \\
\hline Magreza & $35(61,4 \%)$ & $22(38,6 \%)$ & \\
\hline Excesso de peso & $17(68,0 \%)$ & $08(32,0 \%)$ & \\
\hline Amenorreia no $1^{\circ}$ atendimento $(n=185)$ & & & 0,289 \\
\hline Sim & $42(58,3 \%)$ & $30(41,7 \%)$ & \\
\hline Não & $79(69,9 \%)$ & $34(30,1 \%)$ & \\
\hline Amenorreia no último atendimento $(n=138)$ & & & 0,068 \\
\hline Sim & $17(85 \%)$ & $03(15 \%)$ & \\
\hline Não & $64(54,2 \%)$ & $54(45,8 \%)$ & \\
\hline
\end{tabular}

Tabela 4. Idade e dados do seguimento dos pacientes que abandonaram (GA: $n=156$ ) e que não abandonaram (GNA: $n=$ 78) o tratamento - Ribeirão Preto-SP, 2013

\begin{tabular}{|c|c|c|c|c|}
\hline & Média & Mínimo & Máximo & $\begin{array}{l}\text { Desvio- } \\
\text { padrão }\end{array}$ \\
\hline \multicolumn{5}{|c|}{ Idade (anos): } \\
\hline $\mathrm{GA}$ & 20,5 & 10 & 41 & 6,88 \\
\hline GNA & 22,1 & 12 & 78 & 10,25 \\
\hline \multicolumn{5}{|c|}{ Tempo de sintomas (meses): } \\
\hline$G A$ & 48,72 & 02 & 324 & 57,28 \\
\hline GNA & 49,01 & 04 & 480 & 77,05 \\
\hline \multicolumn{5}{|c|}{ IMC no $1^{10}$ atendimento $\left(\mathrm{kg} / \mathrm{m}^{2}\right)$ : } \\
\hline $\mathrm{GA}$ & 18,95 & 9,0 & 42,0 & 5,06 \\
\hline GNA & 17,16 & 8,0 & 45,0 & 5,31 \\
\hline \multicolumn{5}{|c|}{ IMC no último atendimento $\left(\mathrm{kg} / \mathrm{m}^{2}\right)$ : } \\
\hline GA & 20,48 & 12,0 & 42,0 & 4,61 \\
\hline GNA & 20,86 & 11,0 & 45,0 & 5,41 \\
\hline \multicolumn{5}{|c|}{$\begin{array}{l}\text { Tempo de amenorreia no } 1^{\circ} \text { atendimento } \\
\text { (meses): }\end{array}$} \\
\hline GA & 3,84 & 0 & 144 & 14,18 \\
\hline GNA & 5,86 & 0 & 144 & 18,83 \\
\hline \multicolumn{5}{|c|}{$\begin{array}{l}\text { Tempo de amenorreia no último atendimento } \\
\text { (meses): }\end{array}$} \\
\hline GA & 2,57 & 0 & 45 & 6,99 \\
\hline GNA & 0,46 & 0 & 16 & 2,3 \\
\hline
\end{tabular}

IMC: índice de massa corporal.
Tabela 5. Tempo de tratamento dos pacientes que abandonaram $(G A ; n=156)$ e que não abandonaram (GNA; $n=78)$ o tratamento - Ribeirão Preto-SP, 2013

\begin{tabular}{lc}
\hline Tempo de tratamento & $\mathrm{n}(\%)$ \\
\hline$\leq 6$ meses & \\
GA & $81(91 \%)$ \\
GNA & $08(9 \%)$ \\
Total & $89(100 \%)$ \\
Entre 7 e 12 meses & \\
GA & $24(66,7 \%)$ \\
GNA & $12(33,03 \%)$ \\
Total & $36(100 \%)$ \\
$\geq 12$ meses & \\
GA & $50(46,3 \%)$ \\
GNA & $58(53,7 \%)$ \\
Total & $108(100 \%)$ \\
\hline
\end{tabular}

Tabela 6. Estimativas dos parâmetros do modelo de regressão logística - Ribeirão Preto-SP, 2013

\begin{tabular}{lcccc}
\hline Parâmetros & Estimativas & Erro-padrão & Z-value & p-valor \\
\hline Primeiro atendimento: & & & & \\
1980 a 1989 & 3,503 & 0,855 & 16,783 & $<0,05$ \\
1990 a 1999 & 2,674 & 0,675 & 15,688 & $<0,05$ \\
2000 a 2009 & 2,862 & 0,656 & 19,043 & $<0,05$ \\
2010 a 2013 & & & 21,592 & $<0,05$ \\
Presença de comorbidades clínicas & $-0,850$ & 0,371 & 5,258 & $<0,05$ \\
Tempo de tratament0: & & & & \\
$\quad$ 6 meses & 3,3626 & 0,622 & 33,960 & $<0,05$ \\
6a 12 meses & 1,191 & 0,492 & 5,860 & $<0,05$ \\
>12 meses & & & 34,812 & $<0,05$ \\
Estado nutricional: & & & & \\
$\quad$ Eutrofia & 0 & 0,445 & 3,991 & $<0,05$ \\
$\quad$ Excesso de peso &, 889 & 0,628 & 3,467 & 0,063 \\
$\quad$ Magreza & 1,169 & & 4,844 & 0,089 \\
\hline
\end{tabular}

Tabela 7. Análise de regressão logística com o desfecho Abandono como variável dependente - Ribeirão Preto-SP, 2013

\begin{tabular}{lccc}
\hline & OR & $2,5 \%$ & $97,5 \%$ \\
\hline Primeiro atendimento: & & & \\
$\quad$ 1980 a 1989 & 33,208 & 6,215 & 177,437 \\
1990 a 1999 & 14,499 & 3,861 & 54,453 \\
2000 a 2009 & 17,491 & 4,837 & 63,241 \\
Presença de comorbidades clínicas & 0,428 & 0,207 & 0,884 \\
Tempo de tratamento: & & & \\
$\quad<6$ meses & 37,573 & 11,097 & 127,221 \\
6 a 12 meses & 3,289 & 1,254 & 8,625 \\
Estado nutricional: & & & \\
$\quad$ Eutrofia & 2,433 & 1,017 & 5,823 \\
$\quad$ Excesso de peso & 3,219 & 0,940 & 11,021 \\
\hline
\end{tabular}

\section{DISCUSSÃO}

Os resultados apontaram para uma associação estatisticamente significativa entre o diagnóstico do TA e o abandono do tratamento, e os que abandonaram tinham AN. Vários trabalhos que investigaram ambos os TA referem que pacientes com AN, em especial a do tipo compulsivo-purgati- 
vo, são os que mais abandonam o seguimento ${ }^{16,18,23}$. Bandini et al. ${ }^{17}$, ao investigarem essa temática, encontraram que $60 \%$ dos pacientes com AN estavam nessa condição.

Em contrapartida, Swan-Kremeier et al. ${ }^{24}$ encontraram maiores taxas de abandono entre pacientes com BN e TASOE e defendem que a razão pela qual o índice foi menor nos pacientes com diagnóstico de AN pode ser devido a maior gravidade desses casos, do ponto de vista antropométrico, fazendo com que eles reconheçam mais a importância do tratamento. De forma semelhante, Schnicker et al. ${ }^{19}$, ao investigarem o abandono da psicoterapia no tratamento para TA, observaram menor proporção desse evento em pacientes com AN. Eles justificaram o achado com o fato de tais pacientes tenderem a ter a vida cotidiana mais negativamente afetada pelos sintomas e permanecerem no tratamento devido ao estresse e sofrimento causados pela doença. Outra razão é que, participando do tratamento ambulatorial, eles evitariam a hospitalização e, portanto, a motivação para continuar o seguimento em regime ambulatorial seria maior. Nessa mesma direção, Crisp et al. ${ }^{25}$ mostraram que os pacientes com AN aos quais foi oferecido aleatoriamente o tratamento nas modalidades de internação e ambulatorial, geralmente, preferiam o regime ambulatorial.

Swan-Kremeier et al. ${ }^{24}$ e Morlino et al. ${ }^{26}$, ao avaliarem pacientes na modalidade de tratamento ambulatorial diagnosticados com AN, BN e TASOE, encontraram que $73,4 \%$ e $53 \%$, respectivamente, abandonaram o seguimento. Entretanto, outros estudos encontraram índices amplos variando entre $11 \%$ e $45 \% 15,23,27-29$. Tal variabilidade parece ser influenciada pelo tipo de diagnóstico investigado e pelo tratamento oferecido. Hoste et al. ${ }^{30}$ sugerem que, em estudos em que as taxas de abandono sejam menores, os autores deveriam descrever seus esforços específicos para manter os pacientes no tratamento, pois, ao partilhar tais informações, podem contribuir com os serviços de atendimento para TA e também para a concepção de novos estudos sobre o tratamento.

Nesta pesquisa, as características sociodemográficas dos pacientes não influenciaram o abandono. A maioria dos participantes residia em Ribeirão Preto e região, resultado similar ao da pesquisa de Carvalho ${ }^{18}$, que avaliou os fatores de risco para o abandono do tratamento hospitalar para TA de um serviço especializado na cidade de São Paulo. Ambos os grupos (abandono ou não) residiam na mesma cidade onde se localizava o serviço de saúde estudado, mostrando que o abandono do tratamento não sofreu influência dessa variável.

Apesar de o estado civil não ter apresentado significância estatística com o abandono, notou-se que a maioria dos pacientes do GA era solteira, seguida pelos casados e separados. Swan-Kremeier et al..$^{24}$ também não encontraram associação entre essas variáveis, e a maioria dos pacientes que abandonaram o tratamento era solteira.

O estado civil dos participantes desse estudo pode ter relação com a idade deles, uma vez que no GA ela foi de
20,5 anos, revelando que eles eram adultos jovens. Resultados similares foram observados por Fassino et al. ${ }^{16}$, cuja idade média dos pacientes que abandonaram o tratamento foi de 24,7 anos para aqueles em regime de internação e de 23,9 anos para os alocados em regime ambulatorial. Outros estudos também encontraram faixa etária no Grupo Abandono entre 20 e 30 anos $^{15,19,31-34}$. Embora os achados apontem que os que abandonam o tratamento geralmente estão na faixa etária jovem, a idade mais avançada no início do tratamento, juntamente com fatores relacionados à doença como preocupações com o peso, restrição alimentar e medos, psicopatologia geral e baixo nível escolar, é citada como preditora do abandono ${ }^{35,36}$.

A escolaridade dos participantes deste estudo apontou que a maioria dos que abandonaram o tratamento tinha o nível fundamental de ensino completo. Catellani et al. ${ }^{29}$, ao pesquisarem possíveis fatores de risco para o abandono do tratamento de TA na Itália, também não encontraram relação entre a escolaridade e o abandono, concluindo que $58,7 \%$ dos que abandonaram tinham nível médio de ensino.

Quanto à profissão, a maioria do GA deste estudo era estudante. Schnicker et al. ${ }^{19}$ encontraram no grupo que abandonou o tratamento que $21,9 \%$ dos que tinham AN e 39,7\% dos que tinham BN estavam empregados ou eram estudantes.

Em relação à época do seguimento, identificou-se que o primeiro e o último atendimento dos pacientes se deram nos anos 2000 (entre 2000 e 2009), período em que o serviço recebeu a maior demanda de casos. Acredita-se que a procura foi crescendo ao longo dos anos devido ao melhor esclarecimento e divulgação em veículos de comunicação de casos de TA e capacitação dos profissionais para o diagnóstico, com reconhecimento desses quadros como doenças graves. Observou-se, ainda, que pacientes que tiveram o primeiro atendimento na década de 1980 possuem 33,2 vezes mais chances de abandonar o tratamento que aqueles atendidos entre os anos de 2010 e 2013. O tratamento na década de 1980 já contava com equipe multidisciplinar com psiquiatra, nutrólogo e nutricionista em atendimento ambulatorial, além do serviço de internação. Com o passar dos anos, o serviço foi se aperfeiçoando e, na década de 1990, psicólogos passaram a fazer parte da equipe. Nos anos 2000, a implantação de grupos de orientação aos familiares e pacientes enriqueceu os atendimentos, e tal estrutura permanece até os dias atuais ${ }^{21,37}$. Além disso, a equipe vivenciou experiências de multi- e interdisciplinaridade, explorando, nos seus recursos humanos, modos de enfrentamento e autocuidado ${ }^{38,39}$. Tais modificações no padrão de atendimento ao longo dos anos podem ter relação com as menores chances de o paciente abandonar o tratamento, aliado à participação ativa da família nos grupos de apoio, o que poderia gerar maior motivação no seguimento.

O tempo de sintomas dos pacientes do GA deste estudo foi bem variável e apresentou média de quatro anos ( $D P=$ 
4,7), resultado semelhante ao do estudo de Fassino et al. ${ }^{31}$, que encontrou duração média dos sintomas de 3,7 anos (DP $=3,4$ ). Dados tão variáveis podem ter sido observados devido a alguns valores discrepantes nas amostras. Entretanto, outros estudos apontam para uma média maior em sintomatologia da doença, como o de Sly et al..$^{34}$, que apresentou média de 10,6 anos ( $D P=8,2$ ), e o de Catellani et al. ${ }^{29}$, cuja média foi de 8,7 anos ( $\mathrm{DP}=5,9$ ).

O estado nutricional no início do tratamento dos pacientes do GA do presente estudo apresentou associação estatisticamente significativa com o abandono, sugerindo que pacientes com eutrofia possuem maiores chances de abandonar o tratamento. Em estudo prospectivo realizado na Espanha, avaliou-se o abandono do tratamento para TA com acompanhamento dos pacientes durante dois anos. Dos 196 indivíduos que iniciaram o seguimento, 49 (32,4\%) abandonaram, e o estado nutricional deles também era de eutrofia $\left(24,7 \mathrm{~kg} / \mathrm{m}^{2}\right)^{40}$. No presente estudo, observou-se ainda que o GNA apresentou estado nutricional de magreza no início do tratamento, o que pode ter colaborado para a permanência desses pacientes no seguimento.

A ausência de comorbidades clínicas na amostra deste estudo se associou ao abandono do tratamento, contrariamente aos achados de Schnicker et al. ${ }^{19}$, ao avaliarem pacientes com AN e BN. Observou-se, ainda, que havia menor incidência de osteopenia entre os que abandonaram o seguimento, porém sem estudos que pudessem ser comparados. Sugere-se que a ausência de osteopenia, bem como a de comorbidades clínicas em geral, se associa ao abandono.

Algumas comorbidades psiquiátricas, como a depressão, têm sido relacionadas ao abandono do tratamento ${ }^{41}$, o que foi observado no presente estudo. Bandini et al. ${ }^{17}$ referem que ter um segundo diagnóstico psiquiátrico pode ser fator de risco para o abandono, pois encontraram relação entre essas variáveis, principalmente a depressão. Zeeck et al. ${ }^{42}$ defendem a ideia de que pacientes com diagnóstico de AN e depressão podem ter mais consciência da necessidade de ajuda e, assim, aceitam melhor o tratamento do que aqueles que não têm depressão. A discrepância entre esses estudos talvez possa ser explicada pelo fato de neste último a amostra ser com pacientes com AN, enquanto Bandini et al. ${ }^{17}$ incluíram outros diagnósticos. Jordan et al. ${ }^{43}$ encontraram alta prevalência dessa situação entre indivíduos que abandonaram o seguimento, sendo o transtorno de personalidade esquiva o que mais contribuiu, com 50\% de prevalência. Outros estudos também encontraram associação entre o abandono do tratamento para TA e transtornos de personalidade ${ }^{31,44,45}$.

\section{CONCLUSÕES}

A taxa de abandono do serviço estudado é alta, e a ausência de comorbidade clínica e o estado nutricional adequado fo- ram associados ao abandono. Os pacientes que abandonaram o tratamento eram adultos jovens, tinham o diagnóstico de AN e longo tempo de sintomas antes do início do tratamento, eram eutróficos e estavam há menos de seis meses no seguimento.

Estudos de caráter retrospectivo podem apresentar algumas limitações e, no presente estudo, o fato de a coleta de dados ter sido realizada com base em prontuários médicos é uma delas. Essas questões se referem à falta de registro de alguns dados dos pacientes, como informações relativas à amenorreia, um importante sinalizador clínico e de presença frequente em pacientes com AN. A amostra investigada, apesar de ter incluído todos os pacientes do serviço para uma análise mais ampla, gerou heterogeneidade das variáveis e limitação ao incluir pacientes que receberam abordagem em nível ambulatorial e de internação, visto que as condições de tratamento são distintas. No entanto, permitiu uma investigação abrangente e inédita do primeiro serviço especializado em TA no país, elucidando alguns fatores associados ao abandono que podem ajudar na identificação prévia de grupos de risco e na implementação de medidas que visem a um melhor prognóstico dessas doenças.

Por fim, estudos prospectivos e baseados em evidências poderão contribuir para as pesquisas dirigidas especificamente ao abandono do tratamento de pacientes com TA, buscando melhor compreensão dessas doenças e seu tratamento.

\section{CONTRIBUIÇÕES INDIVIDUAIS}

Ana Paula Leme de Souza - Foi responsável pela concepção e pelo desenho do estudo; coleta, organização, análise e interpretação dos dados; revisão da literatura e escrita do artigo.

Rosane Pilot Pessa - Foi responsável pela concepção e pelo desenho do estudo, interpretação dos resultados e revisão crítica do conteúdo do artigo.

\section{CONFLITOS DE INTERESSE}

Os autores não possuem conflitos de interesse a serem declarados.

\section{AGRADECIMENTOS}

Os autores agradecem à Coordenação de Aperfeiçoamento de Pessoal de Nível Superior (Capes) pelo apoio financeiro ao primeiro autor deste artigo. 


\section{REFERÊNCIAS}

1. Qian J, Hu Q, Wan Y, Li T, Wu M, Ren Z, et al. Prevalence of eating disorders in the general population: a systematic review. Shanghai Arch Psychiatry. 2013;25(4):212-23.

2. Morgan CM, Vecchiatti IR, Negrão AB. Etiologia dos transtornos alimentares: aspectos biológicos, psicológicos e socioculturais. Rev Bras Psiquiatr. 2002;24(Supl 3):18-23.

3. Fairburn CG, Cooper Z, Doll HA, Welch SL. Risk factors for anorexia nervosa: three integrated case-control comparisons. Arch Gen Psychiatry. 1999;56(5):468-76.

4. Torre AM. Nutrición y metabolismo en transtornos de la conducta alimentaria. Barcelona: Glosa; 2004.

5. Pinheiro AP, Raney TJ, Thornton LM, Fichter MM, Berrettini WH, Goldman D, et al. Sexual functioning in women with eating disorders. Int J Eat Disord. 2010;43(2):123-9.

6. Sicchieri JMF, Bighetti F, Borges NJBG, Santos JE, Ribeiro RPP. Manejo nutricional dos transtornos alimentares. Medicina (Ribeirão Preto). 2006;39(3):371-4.

7. Claudino AM, Zanella MT. Guias de medicina ambulatorial e hospitalar - Unifesp/Escola Paulista de Medicina - Transtornos alimentares e obesidade. São Paulo: Manole; 2005. $321 p$.

8. Pinzon V, Gonzaga AP, Cobelo A, Labaddia E, Belluzzo P, Fleitlich-Bilyk B. Peculiaridades do tratamento da anorexia e da bulimia nervosa na adolescência: a experiência do PROTAD. Rev Psiquiatr Clín. 2004;31(4):167-9.

9. Cabrera CC. Estratégias de intervenção interdisciplinar no cuidado com o paciente com transtorno alimentar: 0 tratamento farmacológico. Medicina (Ribeirão Preto). 2006:39(3):375-80

10. Treasuare J, Zipfel S, Micali N, Wade T, Stice E, Claudino A, et al . Anorexia nervosa. Nat Rev. 2015:1:1-21.

11. Ribeiro RPP, Bighetti F, Santos MA, Oliveira EA, Unamuno MRDL, Santos JE. Transtornos alimentares: anorexia e bulimia nervosas. In: Kalinowski CE, editor. Programas de atualização em enfermagem: saúde do adulto. Porto Alegre: Artmed; 2006. p. 24-5.

12. Nemeroff CB. Management of treatment-resistant major psychiatric disorders. Oxford: Oxford University Press; 2012.

13. Ratnasuriya RH, Eisler I, Szmukler GI, Russell GF. Anorexia nervosa: outcome and prognostic factors after 20 years. Br J Psychiatry. 1991;158:495-502.

14. Carter JC, Blackmore E, Sutandar-Pinnock K, Woodside DB. Relapse in anorexia nervosa: a survival analysis. Psychol Med. 2004;34(4):671-9.

15. Watson HJ, Fursland A, Byrne S. Treatment engagement in eating disorders: who exits before treatment? Int J Eat Disord. 2013;46(6):553-9.

16. Fassino S, Pierò A, Tomba E, Abbate-Daga G. Factors associated with dropout from treatment for eating disorders: a comprehensive literature review. BMC Psychiatry. 2009;9:67.

17. Bandini S, Antonelli G, Moretti P, Pampanelli S, Quartesan R, Perriello G. Factors affecting dropout in outpatient eating disorder treatment. Eat Weight Disord. 2006;11(4):179-84.

18. Carvalho LSL. Fatores de risco para 0 abandono do tratamento hospitalar para transtornos alimentares [dissertação]. São Paulo: Universidade de São Paulo; 2013.

19. Schnicker K, Hiller W, Legenbauer T. Drop-out and treatment outcome of outpatient cognitive-behavioral therapy for anorexia nervosa and bulimia nervosa. Compr Psychiatry. 2013:54(7):812-23.

20. Santos JE. GRATA: nossa história, trabalho e desafios. Medicina Ribeirão Preto. 2006;39(3):323-6

21. Bighetti F, Santos JE, Ribeiro RPP. Grupo de orientação clínico-nutricional a familiares de portadores de transtornos alimentares: uma experiência "GRATA". Medicina Ribeirão Preto. 2006;39(3):410-4

22. Palma RFM, Santos JE, Ribeiro RPP. Hospitalização integral para tratamento dos transtornos alimentares: a experiência de um serviço especializado. J Bras Psiquiatr. 2013;62(1):31-7.

23. Carter 0, Pannekoek L, Fursland A, Allen KL, Lampard AM, Byrne SM. Increased wait-list time predicts dropout from outpatient enhanced cognitive behaviour therapy (CBT-E) for eating disorders. Behav Res Ther. 2012;50(7-8):487-92.
24. Swan-Kremeier LA, Mitchell JE, Twardowski T, Lancaster K, Crosby RD. Travel distance and attrition in outpatient eating disorders treatment. Int J Eat Disord. 2005;38(4):367-70.

25. Crisp AH, Norton K, Gowers S, Halek C, Bowyer C, Yeldham D, et al. A controlled study of the effect of therapies aimed at adolescent and family psychopathology in anorexia nervosa. Br J Psychiatry. 1991;159:325-33.

26. Morlino M, Di Pietro G, Tuccillo R, Galietta A, Bolzan M, Senatore I, et al. Drop-out rate in eating disorders: could it be a function of patient-therapist relationship? Eat Weight Disord. 2007;12(3):e64-7.

27. le Grange D, Crosby RD, Rathouz PJ, Leventhal BL. A randomized controlled comparison of family-based treatment and supportive psychotherapy for adolescent bulimia nervosa. Arch Gen Psychiatry. 2007;64(9):1049-56.

28. Dejong $H$, Broadbent $H$, Schmidt U. A systematic review of dropout from treatment in outpatients with anorexia nervosa. Int J Eat Disord. 2012;45(5):635-47.

29. Catellani S, Arnone F, De Bernardis E, Ferrari S, Pingani L, Ziosi G, et al. Predictors of dropout from inpatient treatment for eating disorders. Eur Psychiatry. 2011;26:716.

30. Hoste RR, ZaitsoffS, Hewell K, le Grange D. What can dropouts teach us about retention in eating disorder treatment studies? Int J Eat Disord. 2007:40(7):668-71.

31. Fassino S, Daga GA, Pierò A, Rovera GG. Dropout from brief psychotherapy in anorexia nervosa. Psychother Psychosom. 2002;71(4):200-6.

32. Fassino S, Abbate-Daga G, Pierò A, Leombruni P, Rovera GG. Dropout from brief psychotherapy within a combination treatment in bulimia nervosa: role of personality and anger. Psychother Psychosom. 2003;72(4):203-10.

33. Stein KF, Wing J, Lewis A, Raghunathan T. An eating disorder randomized clinical trial and attrition: profiles and determinants of dropout. Int J Eat Disord. 2011:44(4):356-68.

34. Sly R, Mountford VA, Morgan JF, Lacey JH. Premature termination of treatment for anorexia nervosa: differences between patient-initiated and staff-initiated discharge. Int J Eat Disord. 2014;47(1):40-6

35. Woodside DB, Carter JC, Blackmore E. Predictors of premature termination of inpatient treatment for anorexia nervosa. Am J Psychiatry. 2004;161(12):2277-81.

36. Huas C, Godart N, Foulon C, Pham-Scottez A, Divac S, Fedorowicz V, et al. Predictors of dropout from inpatient treatment for anorexia nervosa: data from a large French sample. Psychiatry Res. 2011;185(3):421-6.

37. Santos MA. Sofrimento e esperança: grupo de pacientes com anorexia e bulimia nervosas. Medicina (Ribeirão Preto). 2006;39(3):386-402.

38. Silva LM, Santos MA. Construindo pontes: relato de experiência de uma equipe multidisciplinar em transtornos alimentares. Medicina (Ribeirão Preto). 2006;39(3):415-24.

39. César ABC, Pereira MF, Russo R, Soares ACN, Vicente AT. Multiplicadores reflexivos: uma experiência do Instituto Familiae junto ao Grupo de Assistência em Transtornos Alimentares (GRATA) do HC-FMRP-USP. Medicina ()Ribeirão Preto. 2006:39(3):433-8.

40. Rodríguez-Cano T, Beato-Fernandez L, Moreno LR, Vaz Leal FJ. Influence of attitudes towards change and self-directness on dropout in eating disorders: a 2-year follow-up study. Eur Eat Disord Rev. 2012;20(3):e123-8.

41. Steel Z, Jones J, Adcock S, Clancy R, Bridgford-West L, Austin J. Why the high rate of dropout from individualized cognitive-behavior therapy for bulimia nervosa? Int J Eat Disord. 2000;28(2):209-14

42. Zeeck A, Hartmann A, Buchholz C, Herzog T. Drop outs from in-patient treatment of anorexia nervosa. Acta Psychiatr Scand. 2005;111(1):29-37.

43. Jordan J, McIntosh VV, Carter FA, Joyce PR, Frampton CM, Luty SE, et al. Clinical characteristics associated with premature termination from outpatient psychotherapy for anorexia nervosa. Eur Eat Disord Rev. 2014;22(4):278-84.

44. Nozaki T, Motoyama S, Arimura T, Morita C, Koreeda-Arimura C, Kawai K, et al. Psychopathological features of anorectic patients who dropped out of inpatient treatment as assessed by the Minnesota multiphasic personality inventory. Biopsychosoc Med. 2007;1:15.

45. Pham-Scottez A, Huas C, Perez-Diaz F, Nordon C, Divac S, Dardennes R, et al. Why do people with eating disorders drop out from inpatient treatment?: the role of personality factors. J Nerv Ment Dis. 2012;200(9):807-13. 